\title{
Synchrotron X-ray tomographic study of a Silicon electrode before and after discharge and the effect of cavities on particle fracturing ${ }^{\#}$
}

\author{
Lukas Zielke $*^{a}$, Fu Sun $^{b}$, Henning Markötter $^{b}$, André Hilger ${ }^{b}$, Riko Moroni $^{a}$, \\ Roland Zengerle ${ }^{a, c}$, Simon Thiele $e^{a, d}$, John Banhart ${ }^{b}$ and Ingo Manke
}

Lukas Zielke, Riko Moroni

a Laboratory for MEMS Applications, IMTEK Department of Microsystems Engineering, University of Freiburg, Georges-Koehler-Allee 103, 79110 Freiburg, Germany

E-mail: Lukas.Zielke@imtek.de, Riko.Moroni@imtek.de

Prof. Dr. John Banhart, Dr. Ingo Manke, Fu Sun, Dr. André Hilger, Dr. Henning Markötter

b Helmholtz Zentrum Berlin, Hahn-Meitner-Platz 1, 14109 Berlin, Germany

E-mail: banhart@helmholtz-berlin.de,manke@helmholtz-berlin.de, fu.sun@helmholtzberlin.de, hilger@helmholtz-berlin.de, markoetter@helmholtz-berlin.de

Prof. Dr. Roland Zengerle

a Laboratory for MEMS Applications, IMTEK Department of Microsystems Engineering,

University of Freiburg, Georges-Koehler-Allee 103, 79110 Freiburg, Germany

c Hahn-Schickard, Georges-Köhler-Allee 103, 79110 Freiburg, Germany

Zengerle@imtek.de

Dr. Simon Thiele

a Laboratory for MEMS Applications, IMTEK Department of Microsystems Engineering,

University of Freiburg, Georges-Koehler-Allee 103, 79110 Freiburg, Germany

d FIT, University of Freiburg, Georges-Köhler-Allee 105, 79110 Freiburg, Germany,

Simon.Thiele@imtek.de

\#published in: ChemElectroChem 3(7), 1170-1177 (2016)

Wiley, ISSN: 2196-0216 — doi: 10.1002/celc.201600219

Silicon ( $\mathrm{Si}$ ) has been proposed as one of the most promising anode materials for next-generation lithium ion batteries (LIBs). However, unsatisfactory discharge capacity/energy density and inevitable performance worsening prevent their commercialization. Herein, an in-depth investigation on the same Si composite electrode before and after the first discharge by employing in situ synchrotron $\mathrm{X}$-ray tomography is presented. It is found that $\mathrm{i}$ ) on the electrode level, the $\mathrm{Si}$ particles located in the central part of the electrode preferentially experience crack formation; ii) on the individual particle level, heterogeneous electrochemical lithiation behaviour is observed; iii) cavities are formed during the electrode preparation and battery operation. Moreover, the correlation between the electrochemical activities of $\mathrm{Si}$ particles and their individual electrical contact to the electron conducting network is investigated. For the first time it is quantified that $\mathrm{Si}$ particles will experience lithiation only under the condition that at least $40 \%$ of their surface is electrically connected. These novel insights are possible explanations for low discharge capacity/energy of $\mathrm{Si}$ electrode LIBs, and would open new design principles and opportunities for high-capacity electrode materials for next-generation energy storage systems. 


\section{Introduction}

Next-generation lithium ion batteries (LIBs) with improved specific power and energy density have been proposed for a variety of demanding applications from electric vehicles to large-scale grid storage facilities. ${ }^{[1]}$ Worldwide efforts are therefore underway to find novel electrode materials that will be considered as alternative options to replace the currently commercialized cathode and anode materials. ${ }^{[2]}$ On this search for high-capacity anode materials, silicon ( $\mathrm{Si}$ ) has been identified as one of the most promising candidates to substitute graphite ${ }^{[3]}$ : In contrast to graphite, where fully lithiated graphite stores one Liion per six carbon atoms through intercalation, fully lithiated Si-anodes can theoretically store four Li-ions per $\mathrm{Si}$ atom by chemical bonding. This leads to larger theoretical capacities up to $3579 \mathrm{mAhg}^{-1}$, which is about 10 times larger than that of graphite anodes, $372 \mathrm{mAh} \mathrm{g}^{-1}$. ${ }^{[4]}$ However, Si anodes exhibit an unsatisfactory discharge capacity/energy density when discharged using high currents. They additionally show irreversible capacity fading during cycling. Previous studies, employing various characterization tools such as scanning electron microscope (SEM) ${ }^{[5]}$, transmission electron microscope (TEM) ${ }^{[6]}$ and $\mathrm{X}$-ray tomography ${ }^{[7]}$, have suggested that the inevitable capacity decay is associated intimately with the dramatic volume change of $\sim 300 \%$ during cycling, with preferential de/lithiation pathway along the [110] direction of crystalline $\mathrm{Si}^{[8]}$ In addition, it was found that a two-phase boundary between a crystalline Si core and an amorphous Li-Si shell induces stress within the anode particles, resulting in significant particle fracture and pulverisation. ${ }^{[6,7,9]}$

Apart from boundary-induced fracture mechanisms, other factors also contribute to the decrease in energy density and the irreversible capacity fading. The central issue is related to the weakening or even complete loss of electrical contact between Si particles and the conductive network, consisting of carbon binder domain (CBD) and current collector. ${ }^{[10]}$ Insufficient contact to the conductive network leads to partial electrochemical inactivation of these active materials, resulting in a reduced utilization of loaded material and unsatisfactory dis/charge capacity retention. Unfortunately, extensive characterizations of the evolution of a complete Si based electrode within an operational LIB have not been conducted due to i) the incompatibility between conventional investigation tools and commercial LIBs ${ }^{[11]}$ and ii) the inability to characterize ensembles of active particles contained within a realistic multi-particle electrode. ${ }^{[11]}$ Furthermore, no experimental framework of investigating the lower energy/capacity caused from insufficient contact between active materials and CBD has been reported, although it shows that the rate performance of a LIB electrode composite can be markedly improved by providing alternative electron paths. ${ }^{[12]}$

Here, we report the imaging of the same Si based electrode in pristine and the firstly discharged state in three-dimensions using in situ synchrotron X-ray tomography. Using high fluxes from synchrotron sources, ${ }^{[13]}$ this characterization technique allows us to obtain large and representative datasets to track the evolution of the entire Si electrode on the electrode level (millimetres) and on the level of individual particles $(\sim 5 \mu \mathrm{m})$ simultaneously. Studies on the de/lithiation of Si and other Li-alloying metals (e.g. Sn and Ge) using X-ray tomography/microscopy were conducted in the past ${ }^{[7,11,14-16]}$, in which volume expansions/contractions, the relation between $\mathrm{X}$-ray absorption and lithiation as well as fracturing mechanisms were investigated. Specifically, Taiwo et al. suggested that mechanical stress generated from the significant volume changes can decrease the contact of single Si particles to the conducting network. ${ }^{[16]}$

By employing in-situ synchrotron X-ray tomography, we observed what Taiwo et al. suggested and expanded this theory by a further quantitative analysis. Other important 
findings were i) on the electrode level, the Si particles located in the central part of the electrode preferentially experienced crack formation compared with the Si particles located in the peripheral region. And ii) on the individual particle level, heterogeneous electrochemical lithiation behaviour was observed compared with the widely employed macro-homogeneuos model for battery simulation. ${ }^{[17]}$ Finally, we quantified the fracturing of Si particles and related particles that remain unfractured with a loss of electrical/ionic contact to either the electron conductive network (current collector or to the carbon binder domain (CBD)) or the $\mathrm{Li}$ ion conductive electrolyte. Possible reasons for the contact loss have also been proposed. The current study fundamentally expands the inherent explanations for low discharge capacity/energy of Si electrode LIBs, and would open new design principles and opportunities for high-capacity electrode materials for nextgeneration energy storage systems.

\section{Results}

\subsection{Morphology of the Pristine Electrode and Electrochemical Characterization}

$\mathrm{X}$-ray tomography is based on measuring variations in X-ray attenuation coefficients in a rotating sample and three-dimensional (3D) reconstructions of samples with high spatial resolution can be obtained by using high-flux synchrotron X-ray facilities. ${ }^{[18]}$ Since all phases present (Si particles, CBD and cavity) exhibited excellent contrast due to different $\mathrm{X}$-ray attenuation coefficients, morphological changes of particles, local CBD destruction and cavity formation could be studied in detail in 3D.

In the present study, a proof-of-concept battery, fully compatible with a synchrotron X-ray tomography setup (Figure 1a and b) and simultaneously representative of commercial LIBs, was used. We focused on morphological changes in the entire Si composite electrode before and after the first discharge. The experimental setup is shown in Figure 1a. The custom made cell allows imaging all inner components within the proof-of-concept battery (Figure 1b). Before starting the in-situ X-ray tomography we conducted scanning electron microscope (SEM) characterization of the prepared Si composite electrode surface for a reliable interpretation of the X-ray tomography data. An exemplary image is shown in Figure S1: It can clearly be seen, that the Si particles are well mixed with the CBD, which agrees with the tomography data (Figure 1c, inset), where the spherical Si particles can clearly be discriminated from the CBD filled pore space. The nano pores in the CBD cannot be resolved using synchrotron X-ray tomography with a pixel size of $438 \mathrm{~nm}$. ${ }^{[19,20]}$ Therefore, in the following CBD denotes a mixture of small, electrolyte filled pores and carbon black particles glued together with binder.

In a first step, we performed cyclic voltammetry to verify the reduction and oxidation characteristics of the Si particles (Figure S2). The observed anodic/cathodic peaks are in good agreement with those of previously reported silicon/carbon/binder (Si/C) composites ${ }^{[2,21]}$. Subsequently, a tomography in the pristine state (without any cycling) was made for reference. It is shown in Figure 1c: The battery contains a Si/C composite electrode, a separator (24 $\mu \mathrm{m}$ thick) and a lithium metal electrode (as counter and reference electrode). It can clearly be seen that the currently investigated Si/C composite electrode consists of an ensemble of active particles hold together by the electron conducting CBD, as in most realistic electrodes. 

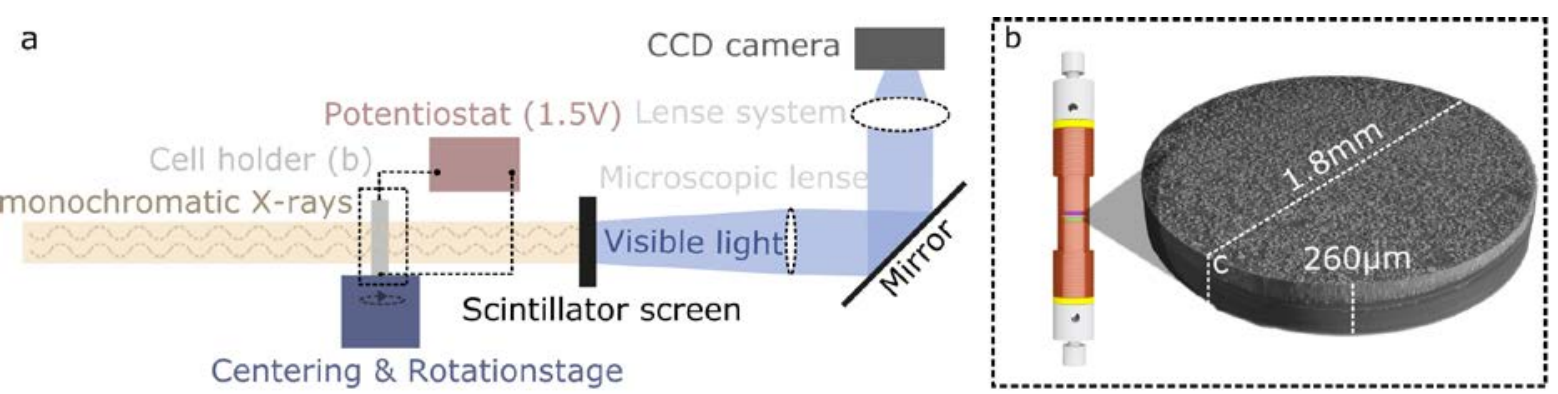

Centering \& Rotationstage

C

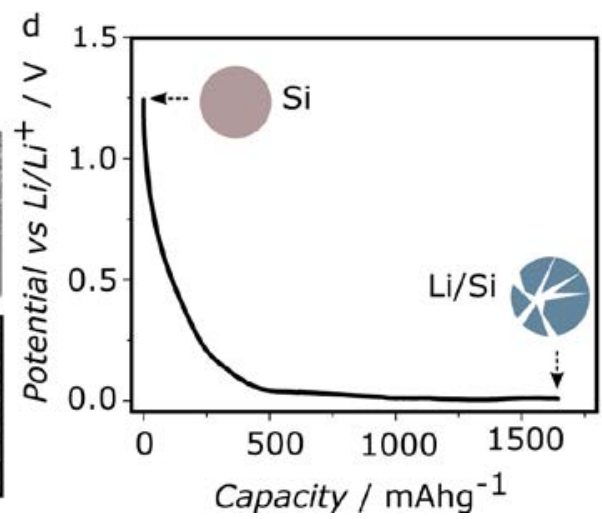

Figure 1 a) Schematic illustration of experimental setup at the BAMline at the electron storage ring BESSY II in Berlin, Germany. b) Battery cell holder design. c) Cell with lithium metal (bottom), separator (24 $\mathrm{m}$, centre) and a silicon/carbon/binder electrode (top). The lithium flux during discharge is indicated by arrows. An exemplary part of the electrode is shown in the inset. The scale bar represents $50 \mu \mathrm{m}$. d) First discharge curve of the battery at $0.13 \mathrm{Ag}^{-1}$, where the schematic sketch of an intact Si particle represents the pristine state and the cracked Li/Si particle represents the discharged state.

After the tomography in the pristine state, the battery was discharged at $0.13 \mathrm{Ag}^{-1}(\sim 0.03 \mathrm{C})$ based only on the mass of the loaded Si. The corresponding discharge curve is shown in Figure 1d. The obtained specific capacity was around $1700 \mathrm{mAhg}^{-1}$, which is lower than the theoretical specific capacity of Si (3579 $\left.\mathrm{mAhg}^{-1}\right)$. In order to find explanations for the low specific capacity of the Si electrode, another tomography of the same electrode was conducted.

\subsection{Morphology of the Discharged Electrode}

\subsubsection{Heterogeneous Lithiation on the Electrode Level}

The firstly discharged state tomography is shown in Figure 2a, b and c. Compared with the pristine state tomography (Figure 1c), it can be unambiguously discerned that Si particles located in the central part of the electrode preferentially undergo crack formation, denoted by a blue circle in Figure 2b. 

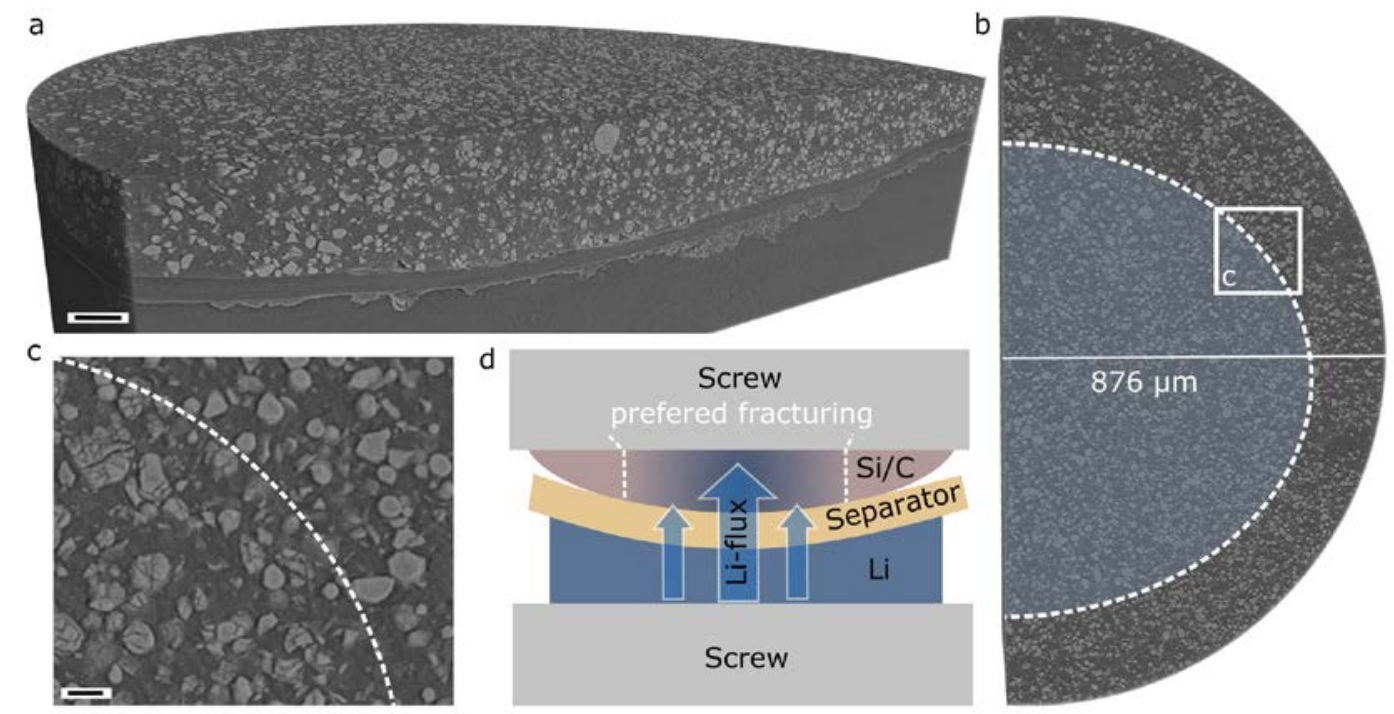

Figure 2 a) Three-dimensional representation of the discharged $\mathrm{Si} / \mathrm{C}$ composite electrode in through-plane direction. The scale bar represents $50 \mu \mathrm{m}$. b) Top-view of the half-cropped reconstruction, showing the region where Si particles fractured preferentially (blue circle). c) Zoom in on the border of the region with preferred fracturing. The scale bar represents $10 \mu \mathrm{m}$. d) Schematic illustration showing the $1 \mathrm{D} \mathrm{Li}$ ions flow paths in the inner part of the Si/C composite electrode.

In contrast, particles located in the peripheral regions are kept intact during the whole discharge process (hereafter, we denote the fractured Si particles as electrochemically active particles or lithiated particles; the unfractured Si particles as electrochemically inactive particles or un-lithiated particles). This finding was further checked by employing two-dimensional particle size distributions, shown in Figure S3. In the region with preferred fracturing (blue circle Figure 2b), the particle size is systematically smaller, confirming the suggestion from the visual analysis of the tomographic dataset. The observed inhomogeneity of lithiation on the entire electrode level corresponds well with previous reports, ${ }^{[22]}$ in which, Cai et al. observed an significant inhomogeneous deterioration in a commercial LIB by using in situ neutron diffraction. They found that near the edges of the battery, both the graphite anode and the spinel-based cathode showed a decreased capacity while near the central area, both electrodes functioned properly. They propose some potential factors, such as the electrolyte solution loss, separator pore clogging and non-uniform temperature can contribute to this phenomenon. In our case, we propose that an inhomogeneous $\mathrm{Li}$ ion flux resulting from an inhomogeneous pressure subjected on $\mathrm{Si} / \mathrm{C}$ composite electrode cause the inhomogeneous lithiation on the electrode level. As schematically shown in Figure 2d, owning to a convex shape of the $\mathrm{Si} / \mathrm{C}$ electrode, an inhomogeneous pressure (a larger pressure in the inner part of the electrode compared with a smaller pressure at the outer parts) can be generated when screwing the $\mathrm{Si} / \mathrm{C}$ electrode and Li electrode together using the two current collector pins (Figure $1 \mathrm{~b}$ ). This is further evidenced by the curvature of the separator between the two electrodes (Figure 1c and 2a). Assuming a homogeneous and electrically well conducting CBD ${ }^{[23]}$, the lithiation process only occurs in regions with strongly promoted $\mathrm{Li}$ conduction pathways. ${ }^{[24]}$ Considering that the flow of Li ions, driven by the electrochemical potential between the $\mathrm{Li}$ electrode and $\mathrm{Si}$ electrode, is faster in the central part (shorter distances) than that of the outer part of $\mathrm{Si} / \mathrm{C}$ electrode (longer distances), the currently observed welldefined one dimensional (1D) Li ions pathway (indicated by arrow in Figure 2d) agrees well with previous reports. ${ }^{[24]}$ Therefore it is reasonable that Si particles located along the 1D Li ions pathway experience significant crack formation (lithiation) while others, located outside the pathway, are kept intact. A direct detrimental effect of the 
inhomogeneous lithiation on the electrode level is that the discharge current subjected on the complete battery $\left(0.13 \mathrm{Ag}^{-1}\right)$ concentrates only on the central part of the $\mathrm{Si} / \mathrm{C}$ electrode, resulting in i) high localized current density and current hotspots that could induce fracture and accelerate capacity fading ${ }^{[25]}$ and ii) significant under-utilization of active materials that could decrease the capacity/energy density. ${ }^{[26]}$ We therefore conclude that it is crucial to design sophisticated electrode architecture that guarantees uniform Li ion flow pathways over the whole electrode.

\subsubsection{Particle Sizes and Volume Fractions}

To further investigate causes leading to low capacity/energy density, a rectangular region of interest (ROI) located within the 1D Li-ion flow pathway was chosen, as defined in Figure S3 and the results are shown in Figure 3. First, we quantified the fracturing of particles in this particular ROI for which we calculated the particle size distribution for the pristine and discharged electrode. As a result, we found a significant thinning of the distribution after discharging the battery, which reflects a general fracturing of particles. The results agree well with previous report. ${ }^{[1]}$ In our case, the fracturing of particles leads to a decreased mean particle diameter from $7 \mu \mathrm{m}$ to $3 \mu \mathrm{m}$ (Figure 3a).
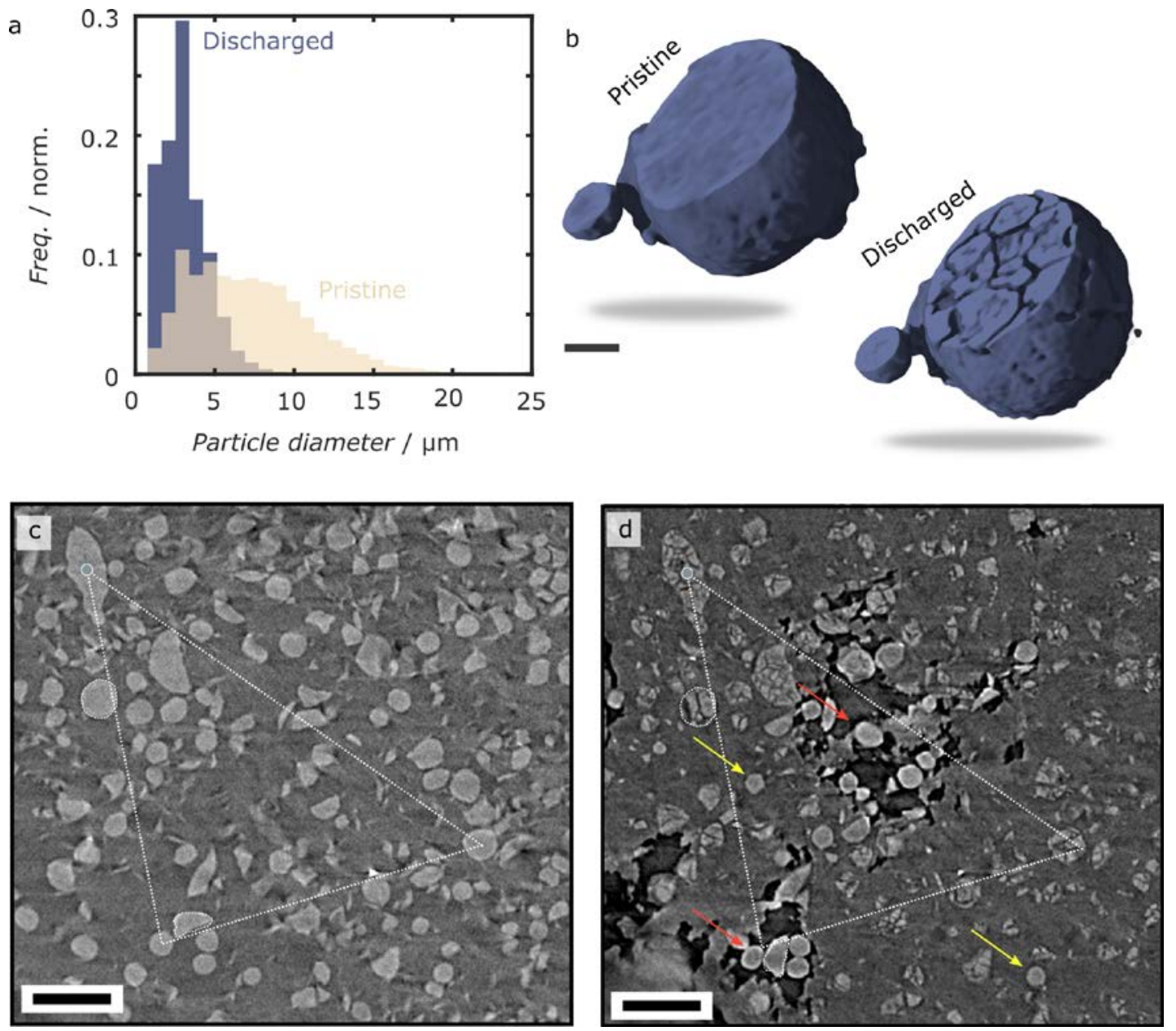

Figure 3 a) Particle size distribution of the pristine (beige) and discharged electrode (blue). b) 3D representation of a large particle, pristine and fractured. c, d) Reconstructed slices of the pristine electrode (c) and the same position after discharge (d) of a small part of the region of interest. The arrows indicate intact particles with good contact to the conducting network (yellow) and bad contact (red). The scale bar is $40 \mu \mathrm{m}$ long. The white triangle is shown for orientation. 
The height of the first bar in the particle size distribution of the discharged electrode in Figure $3 a$ indicates that the size of a certain amount of fractured particles is below the estimated resolution limit of $\sim 1 \mu \mathrm{m}$ ( $\sim 2$ times the pixel size of $438 \mathrm{~nm}$ ). To confirm this, we calculated the Si volume per electrode area. The calculation yielded $31.5 \mu \mathrm{m}^{3} / \mu^{2}$ in the pristine state and $24.0 \mu \mathrm{m}^{3} / \mu^{2}$ in the discharged state, which is a difference of $24 \%$. This difference corresponds very well with another group's value of $25 \%$, which implies a reasonable segmentation. ${ }^{[16]}$ The difference before and after the first discharge indicates that approximately a quarter of the original Si after the first lithiation has fractured into pieces smaller than $1 \mu \mathrm{m}$ in diameter. In Figure 3b a particle before and after discharge is shown for illustration, indicating the fracturing into multiple smaller parts while keeping the originally spherical shape.

\subsubsection{Size and Distribution of Cavities}

Apart from particle fracture, other phenomena in the ROI are demonstrated in Figure 3c and d. Here, the same in-plane region of the electrode is shown in the pristine (Figure 3c) and discharged state (Figure 3d). The white triangle shows that there is little in-plane distortion, even though most of the particles exhibit strong fracturing. The limited volume expansion during the first discharge process suggests limited lithium uptake/storage ability of Si particles. ${ }^{[22]}$ Moreover, it can be observed that some Si particles still undergo no lithiation (no crack formation, indicated by yellow arrows in Figure 3d) even though they are located within the $1 \mathrm{D} \mathrm{Li}$ ions flow pathway, with CBD surrounding them. Actually, the observed inhomogeneous lithiation behaviour among individual particles is in agreement with previous reports that uncharged $\mathrm{FePO}_{4}$ phases are often surrounded by charged $\mathrm{LiFePO}_{4}$ phases ${ }^{[27]}$ and that the statistical measure of local "spectroscopic" state-of-charge (SOC) state in an LIB is heterogeneous. ${ }^{[28]}$ It has been suggested that in a realistic commercial LIB electrode, conditions for a homogeneous electrochemical reaction are hardly satisfied since any fluctuations on the electrolyte exposure, electrical contact or crystal defects can result in an inhomogeneous reaction. ${ }^{[29]}$ In the present study we quantitatively investigated the correlation between the electrochemical activities of $\mathrm{Si}$ particles and the influence of their contact to both the ionically conducting electrolyte and the electrically conducting network.

Another worthy observation is that particles in the dark regions in Figure 3d show no or weak fracturing (indicated by red arrows). As a matter of fact, due to the different X-ray absorption coefficients of Si particles, CBD and large pores, we could identify the dark regions as cavities in the CBD. It is already known that gas evolution, which is intimately related with the solid electrolyte interphase (SEI) formation and the electrolyte decomposition, can locally destroy CBD and induce electrolyte displacement. ${ }^{[30,31]}$ Goers et al. have observed gas formed channels during cycling within a LIB by neutron radiography $(\mathrm{NR}){ }^{[32]}$

In the following, we analyse the cavities in 3D in terms of size, distribution and their influences on Si particle lithiation. Results are shown in Figure 4. First, we present the quantitative analysis of the cavities. The mean diameter of the cavities within the complete $\mathrm{Si} / \mathrm{C}$ electrode was found to be $\sim 5 \mu \mathrm{m}$. However, diameters of up to $\sim 18 \mu \mathrm{m}$ were measured as shown in the size distribution in Figure 4a. 

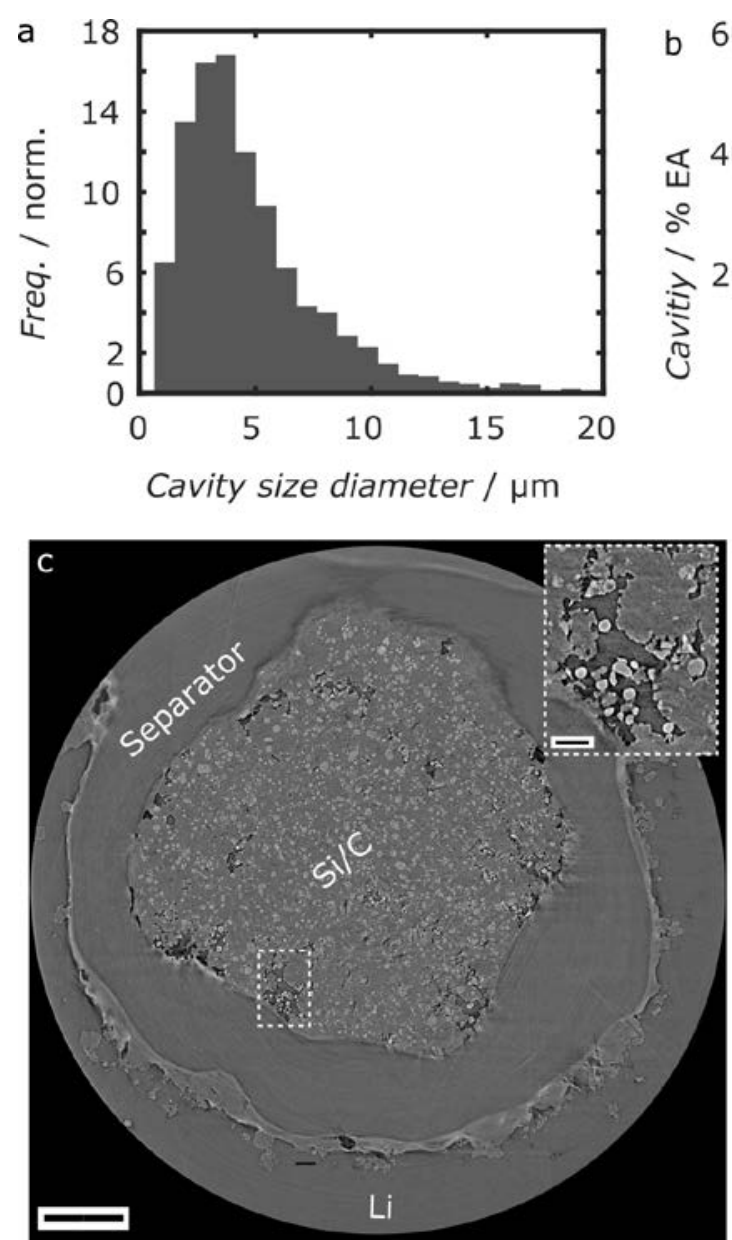

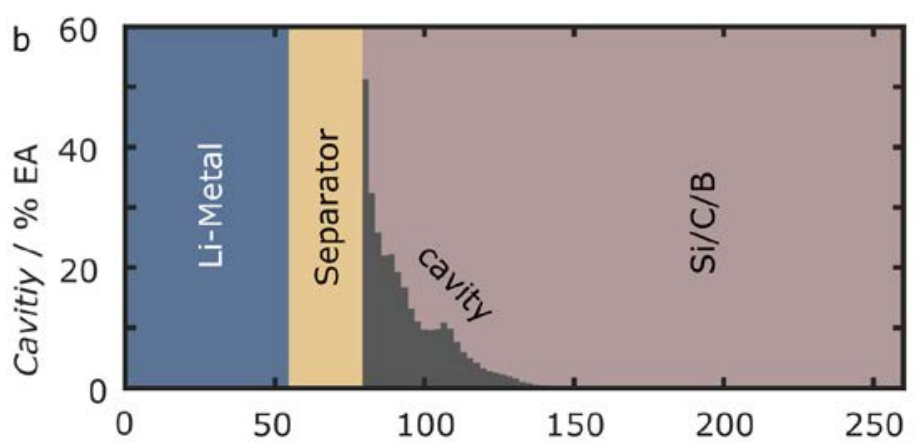

Through-plane height / $\mathrm{mm}$

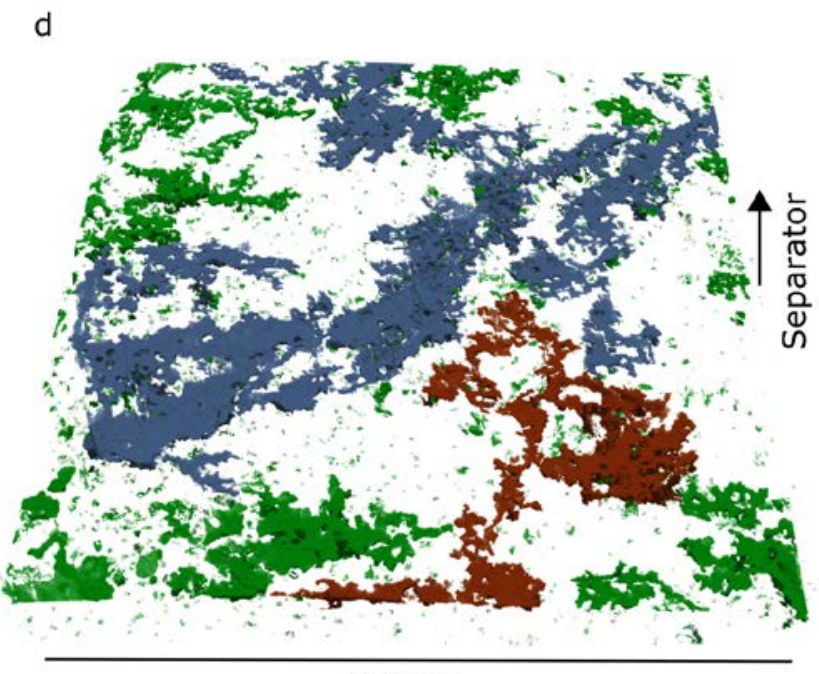

$832 \mu \mathrm{m}$

Figure 4 a) Cavity size distribution in the discharged state. b) Fraction of cavity area per electrode area in the through-plane direction, starting at the separator. c) Exemplary tomogram showing inhomogeneously distributed cavities. One of them is shown in the inset. The scale bars represent $25 \mu \mathrm{m}$ (inset) and $200 \mu \mathrm{m}$. d) Three-dimensional representation of the cavity network. The largest connected cluster is shown in blue and the second largest in red. Remaining cavity clusters are shown in green.

We also found that cavities were mostly located in the vicinity of the separator (Figure 4b) and were not homogeneously distributed throughout the electrode as depicted in Figure 4c (exemplary tomogram) and Figure 4d (3D distributions of cavities). It has to be noted that in Figure 4d, the colours represent connected cavity clusters: the largest connected cavity comprises 63 vol. \% of the total cavity volume (Figure 4d, blue) and spans the whole length of the ROI; the next largest cavity region comprises 17 vol. \% (Figure 4d, red).

\subsubsection{A Correlation between Particle Fracture and Cavities}

Furthermore a quantitative analysis of the influence of cavities on the fracturing of particles in close contact to them was conducted. The results are shown in Figure 5. 


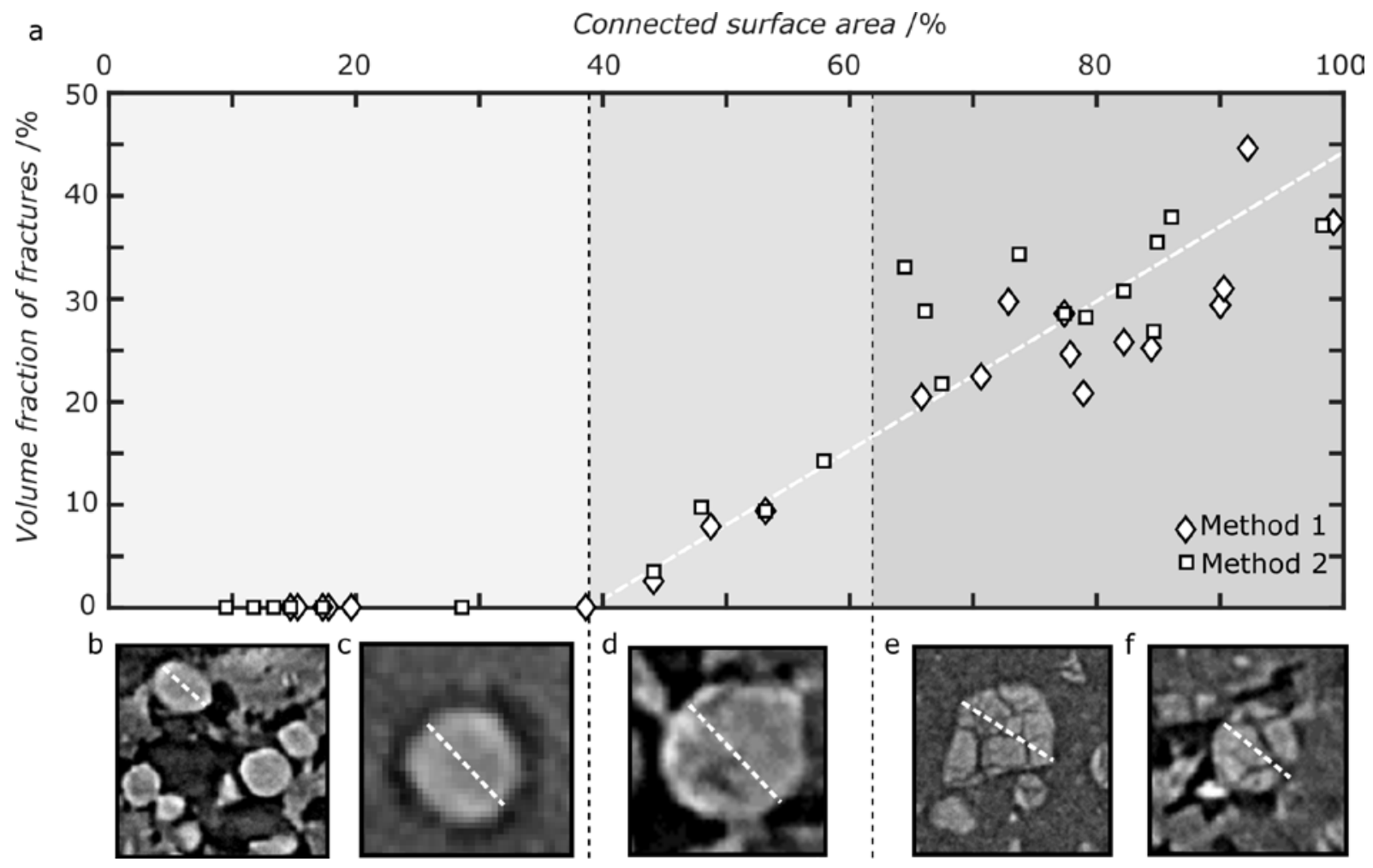

Figure 5 a) Relative volume of fractures within silicon particles versus connected surface area, calculated using two different segmentation methods (diamonds and squares)). b, c) Structurally intact and poorly connected particles: b) within the large cavity network ( shown diameter was $16 \mu \mathrm{m}$ ) and c) with localized CBD destruction around it ( shown diameter was $9 \mu \mathrm{m})$. d) Partially connected particle with a few fractures ( 48\% connected surface area, diameter was $14 \mu \mathrm{m})$. e, f) Well connected Si particle e) with fractures aside of the cavity network ( $90 \%$ connected surface area, diameter of $27 \mu \mathrm{m}$ ) and f) inside of the cavity network ( $77 \%$ connected surface area and a diameter of $14 \mu \mathrm{m}$ ). The white dotted line in a) is a guide to the eye.

Four types of particles were found: i) Particles well connected to the CBD that exhibited large fractures (Figure $5 \mathrm{e}, \mathrm{f}$ ), ii) particles with only partial contact to the CBD but with fractures (Figure $5 \mathrm{~d}$ ), iii) particles with only partial contact to the CBD but without fractures (Figure $5 \mathrm{~b}$ ) and iv) particles almost fully surrounded by a cavity (Figure $5 \mathrm{c}$ ). It needs to be noted that the cavity around the particles in iv) is not part of the large cavity network but is strongly localized around single particles. Actually, this kind of particle (type iv) is representative of a class of Si particles that often undergo no crack formation within the 1D Li ions flow pathway (see Figure 3d). The reason for these strongly localized cavities lies probably in the electrode preparation process, e.g. in the material mixture and/or in the drying process. ${ }^{[33]}$ Further information on type iv particles can be found in the supplementary information.

For a representative quantification of the correlation between particle fracturing and contact loss to both the electrical and ionic network, we investigated 21 different particles in the vicinity or within cavities (both gas generated cavities and localized cavities). Relative volume of fractures within the particles, serving as an indicator for lithiation, versus the electrically/ionically connected surface area of the particles is shown in Figure 5a. We found that, above a threshold of $40 \%$, the degree of fractures intensifies with increasing connected surface area. Since the determination of contact areas is highly critical, all particles and cavities were segmented predominantly manually using two different methods (diamonds and squares), which are described in detail in the Methods section. Despite the scatter of the data a clear trend is observed: the relation between 
fracturing and electrical/ionic contact of Si particles in the battery shows a threshold. That is, a connected surface area below $40 \%$ leads to no electrochemical activity of Si particles. To the authors' knowledge, this is the first investigation on the correlation between the electrochemical activities of Si particles and the influence of their contact area to the conducting electronic/ionic network in the battery.

From an electrochemical point of view, lithium insertion into Si particles during the lithiation process entails Li-ions (from the electrolyte or Li electrode) and electrons (from CBD, conductive network or adjacent active particles) simultaneously. ${ }^{[12]}$ The dependence of the electrochemical performance of LIBs on the contact between active materials and the electrically/ionically conducting network is an important electrode design consideration. ${ }^{[34]}$ Actually, it has been suggested that the electron transference between difference phases is through hopping or tunnelling mechanism with contact resistivity depending exponentially on the gap between the contacts. A gap of 8-10 $\mathrm{nm}$ is large enough to make a contact electrically insulating. ${ }^{[35]}$ Thus, the cavities (either from electrode preparation or battery operation) surrounding Si particles will block the flow of electrons into the active particles during the lithiation process to some extent, resulting in the observed electrochemically in-active (unfractured) particles. After an in-depth quantitative analysis, it is discovered for the first time that, Si particles will experience lithiation only under the condition that at least $40 \%$ of its surface is electronically/ionically connected.

\section{Conclusion}

In summary, we performed an in-situ analysis of a $\mathrm{Si} / \mathrm{C}$ electrode in its pristine and firstly discharged states. Firstly, we found that the Si particles in the centre of the electrode preferentially undergo crack formation while particles located in the peripheral regions are kept intact during the whole first discharge process. We propose that the one-dimensional $\mathrm{Li}$ ions flow behaviour and a non-uniform contact pressure subjected on the $\mathrm{Si} / \mathrm{C}$ electrodes could be the reason. Secondly, we revealed the presence of large non-uniformly distributed cavities (either from electrode preparation or battery operation) within the discharged Si/C electrodes. We further quantified their size and distribution within the electrode in three-dimensions. In addition, an in-depth quantitative analysis was conducted to investigate the dependence of the electrochemical activities of active materials and their contact surfaces to the electrically/ionically conducting network. We found that silicon particles experience fracture only when their contact area to the conducting network is at least $40 \%$ of their total surface area. These results show that synchrotron X-ray tomography is a powerful characterization tool to quantitatively investigate the dependence of the electrochemical performance of LIBs on the electrical contact between active materials and conducting network. The novel insights into possible causes for the lower capacity/energy density of LIBs are crucial because they cannot be easily obtained by conventional macroscopic electroanalytical characterization techniques. Our findings provide potential guidelines to further enhance the performance of LIBs. On the one hand, future electrode engineering should concentrate on optimizing the whole electrode architecture, which can involve all active material particles ionically and electronically connecting to electrolyte and electric conducting network to maximize the utilization of active materials. Particularly, an efficient electrically conducting CBD network and an ionically well-conducting pore space are highly desirable to eliminate the observed electrochemically in-active material. On the other hand, our analysis shows that it is important to develop new electrolytes with reduced gas formation to eliminate gas induced cavities.

\section{Experimental Section}


Materials: Silicon was received from Elkem AS, Norway. Conductive carbon black, Polyvinylidene difluoride (PVDF) binder, Celgard separator and lithium were purchased from MTI Cor. USA. N-methyl pyrrolidone solvent (NMP) and $1 \mathrm{M} \mathrm{LiPF}_{6}$ in a volumeratio mixture (1:1) of ethylene carbonate (EC) and dimethyl carbonate (DMC) were purchased from Sigma Aldrich. The housing of the proof-of-concept beamline battery is made of polyamide-imide (Torlon) from the McMaster-Carr company.

Battery Preparation: The electrode was made of electrode slurries with weight ratios of Si:carbon black:binder of 70:20:10 in NMP. As binder, PVDF was used. Subsequently, the slurry was cast onto an aluminium foil. To remove the NMP, the cast aluminium foils were dried in an oven at $60{ }^{\circ} \mathrm{C}$ overnight. After drying, the completed composite electrode was diced into smaller pieces of around $1.7 \mathrm{~mm} \times 1.7 \mathrm{~mm} \times 0.2 \mathrm{~mm}$ (length $\times$ width $\times$ height) with a razor blade. Before the Si composite was assembled into the battery, it was weighed and the amount of Si particles was determined from the original mass ratio. The mass of the Si composite electrode was $0.17 \mathrm{mg}$. The proof-of-concept battery was assembled in an argon-filled glovebox with humidity and oxygen levels below $0.1 \mathrm{ppm}$. Metallic lithium was placed on the top of a screw, acting as a counter and reference electrode. The polymer separator was placed between the lithium electrode and the Si electrode. Finally, the housing tube was filled with the liquid electrolyte. Current leads were connected to a potentiostat for electrochemical tests.

Electrochemical Measurement: Cyclic voltammetry (CV) and galvanostatic charge/discharge of the battery were carried out with an IviumStat form Ivium Technologies, Netherlerlands. A freshly assembled battery was measured from $2.5 \mathrm{~V}$ to $0 \mathrm{~V}$ at a scan rate of $1 \mathrm{mVs}^{-1}$ to obtain CV curves. During galvanostatic cycling at the beamline, the assembled battery was measured at a discharge current of $0.13 \mathrm{Ag}^{-1}$ based only on the mass of Si active material.

Settings of Tomography Measurements: Synchrotron X-ray tomography was carried out at the BAMline at BESSY II of the Helmholtz-Centre Berlin, Germany. The synchrotron beam was monochromatized to $20 \mathrm{keV}$ using a double multilayer monochromator with an energy resolution of about $1.5 \%$. The detector system comprised a $60-\mu \mathrm{m}$ thick $\mathrm{CdWO}_{4}$ scintillator, a microscopic optic and a pco4000 camera with a $4008 \times 2672$ pixel $^{2}$ CCD chip that is kept out of the direct beam by using a mirror. For tomography, 2200 projections during a $180^{\circ}$ battery rotation, each with $4 \mathrm{~s}$ exposure time were recorded before or after discharge.

Data Processing: The Si/C/B domains were segmented using the software FIJI and applying a median filter and Otsu's method for binarization, followed by a slight opening $[36,37]$. The cavity domain was segmented using the statistical region-merging tool and followed by individual thresholds for subsets. They were chosen by visual judgement. Pore and particle size distributions were calculated using the Delerue method, implemented in the software packages GeoDict (3D global) and Matlab (3D local). The phase distributions (2D and 3D) were calculated using self-programmed Matlab functions. Segmentation of contact areas was conducted using manual segmentation, and two different approaches. Manual segmentation of particles and a global threshold from visual judgement was used to segment the contact area between Si particles and CBD. A dilation of the particle followed, resulting in an overlap of particle and cavity. To avoid edge effects, the segmented particle was eroded once and subsequently the thresholds for the segmentation of fractures were chosen from visual judgment (Method 1). Based on these datasets, the particle-cavity contact area was manually corrected in every main direction, followed by a slight 3D median filter (Method 2).

\section{Supporting Information}


Supporting Information is available from the Wiley Online Library or from the author.

\section{Acknowledgements}

We thank Dr. Heinrich Riesemeier, the beamline scientist at BESSY II, for his valuable assistance, Anna Manzoni for conducting scanning electron microscope (SEM) characterization and Norbert Beck for fabricating the beamline battery. We also thank Elkem AS for providing us with the Si particles. This work is sponsored by the Helmholtz Association and the China Scholarship Council. 


\section{References}

[1] D. S. Eastwood, V. Yufit, J. Gelb, A. Gu, R. S. Bradley, S. J. Harris, D. J. L. Brett, N. P. Brandon, P. D. Lee, P. J. Withers, P. R. Shearing, Adv. Energy Mater. 2014, 4, n/a.

[2] F. Sun, K. Huang, X. Qi, T. Gao, Y. Liu, X. Zou, X. Wei, J. Zhong, Nanoscale 2013, $5,8586$.

[3] D. Larcher, S. Beattie, M. Morcrette, K. Edstrom, J.-C. Jumas, J.-M. Tarascon, J. Mater. Chem. 2007, 17, 3759.

[4] U. Kasavajjula, C. Wang, Appleby, A. John, Selected Papers presented at the FUEL PROCESSING FOR HYDROGEN PRODUCTION SYMPOSIUM at the 230th American Chemical SocietyNational Meeting Washington, DC, USA, 28 August - 1 September 2005 2007, 163, 1003.

[5] S. W. Lee, M. T. McDowell, L. A. Berla, W. D. Nix, Y. Cui, Proceedings of the National Academy of Sciences 2012, 109, 4080.

[6] X. H. Liu, L. Zhong, S. Huang, S. X. Mao, T. Zhu, J. Y. Huang, ACS Nano 2012, 6, 1522.

[7] J. Gonzalez, K. Sun, M. Huang, J. Lambros, S. Dillon, I. Chasiotis 2014, 269, 334.

[8] M. Gu, Y. Li, X. Li, S. Hu, X. Zhang, W. Xu, S. Thevuthasan, D. R. Baer, J.-G. Zhang, J. Liu, C. Wang, ACS Nano 2012, 6, 8439.

[9] M. T. McDowell, S. W. Lee, W. D. Nix, Y. Cui, Advanced Materials 2013, 25, 4966.

[10] C. K. Chan, H. Peng, G. Liu, K. McIlwrath, X. F. Zhang, R. A. Huggins, Y. Cui, Nat Nano 2008, 3, 31.

[11] J. Wang, Y.-c. K. Chen-Wiegart, J. Wang, Angewandte Chemie International Edition 2014, 53, 4460.

[12] G. Chen, T. J. Richardson 2010, 195, 5387.

[13] I. Manke, J. Banhart, A. Haibel, A. Rack, S. Zabler, N. Kardjilov, A. Hilger, A. Melzer, H. Riesemeier, Applied physics letters 2007, 90, 214102.

[14] J. Gonzalez, K. Sun, M. Huang, S. Dillon, I. Chasiotis, J. Lambros 2015, 285, 205.

[15] Weker, J. Nelson, N. Liu, S. Misra, Andrews, J. C., Y. Cui, Toney, M. F., Energy Environ. Sci. 2014, 7, 2771.

[16] O. O. Taiwo, D. S. Eastwood, P. D. Lee, J. M. Paz-García, S. A. Hall, Brett, Daniel J. L, P. R. Shearing, ECS Transactions 2015, 69, 81.

[17] D. Kehrwald, P. R. Shearing, N. P. Brandon, P. K. Sinha, S. J. Harris, J. Electrochem. Soc. 2011, 158, A1393.

[18] F. R. Brushett, L. Trahey, X. Xiao, J. T. Vaughey, ACS Appl. Mater. Interfaces 2014, 6, 4524.

[19] L. Zielke, T. Hutzenlaub, D. R. Wheeler, I. Manke, T. Arlt, N. Paust, R. Zengerle, S. Thiele, Adv. Energy Mater. 2014, 4, 1301617.

[20] L. Zielke, T. Hutzenlaub, D. R. Wheeler, C.-W. Chao, I. Manke, A. Hilger, N. Paust, R. Zengerle, S. Thiele, Adv. Energy Mater. 2015, 5.

[21] X. Zhou, Y.-X. Yin, L.-J. Wan, Y.-G. Guo, Adv. Energy Mater. 2012, 2, 1086.

[22] L. Cai, K. An, Z. Feng, C. Liang, S. J. Harris 2013, 236, 163. 
[23] C. Villevieille, M. Ebner, J. L. Gómez-Cámer, F. Marone, P. Novák, V. Wood, Advanced Materials 2015, 27, 1676.

[24] N. Balke, S. Jesse, Y. Kim, L. Adamczyk, A. Tselev, I. N. Ivanov, N. J. Dudney, S. V. Kalinin, Nano Lett 2010, 10, 3420.

[25] Y. Li, F. El Gabaly, T. R. Ferguson, R. B. Smith, N. C. Bartelt, J. D. Sugar, K. R. Fenton, D. A. Cogswell, Kilcoyne, A. L. David, T. Tyliszczak, M. Z. Bazant, W. C. Chueh, Nat Mater 2014, 13, 1149.

[26] G. Zhang, C. E. Shaffer, C.-Y. Wang, C. D. Rahn, J. Electrochem. Soc. 2013, 160, A2299.

[27] J. Liu, M. Kunz, K. Chen, N. Tamura, T. J. Richardson, J. Phys. Chem. Lett. 2010, 1, 2120.

[28] J. Nanda, J. Remillard, A. O'Neill, D. Bernardi, T. Ro, K. E. Nietering, J.-Y. Go, T. J. Miller, Advanced Functional Materials 2011, 21, 3282.

[29] F. Lin, D. Nordlund, T.-C. Weng, Y. Zhu, C. Ban, R. M. Richards, H. L. Xin, Nat Commun 2014, 5.

[30] E. Sarasketa-Zabala, F. Aguesse, I. Villarreal, Rodriguez-Martinez, L. M., López, C. M., P. Kubiak, J. Phys. Chem. C 2015, 119, 896.

[31] M. Holzapfel, A. Würsig, W. Scheifele, J. Vetter, P. Novák, 13th International Meeting on Lithium Batteries 2007, 174, 1156.

[32] D. Goers, M. Holzapfel, W. Scheifele, E. Lehmann, P. Vontobel, P. Novák, Journal of Power Sources 2004, 130, 221.

[33] D. Mazouzi, Z. Karkar, C. Reale Hernandez, P. Jimenez Manero, D. Guyomard, L. Roué, B. Lestriez, Journal of Power Sources 2015, 280, 533.

[34] B. Orvananos, R. Malik, H.-C. Yu, A. Abdellahi, C. P. Grey, G. Ceder, K. Thornton, Electrochimica Acta 2014, 137, 245.

[35] Nguyen, B. P. N., J. Gaubicher, B. Lestriez, Electrochimica Acta 2014, 120, 319.

[36] M. D. Abràmoff, P. J. Magalhães, S. J. Ram, Biophotonics International 2004, 11, 36.

[37] Nobuyuki Otsu, Trans. Syst., Man, Cybern. 1979, 9, 62. 